\title{
Persistent Systemic Inflammation in Chronic Critical Illness
}

\author{
Christopher E Cox MD MPH
}

\author{
Introduction \\ What Drives the Inflammatory Response in CCI? \\ Clinical Features of Chronic Critical Illness and Their Relationship to \\ Systemic Inflammation \\ Inflammation, Inflammatory Markers, and CCI \\ Inflammation Seen in Precipitants of CCI May Lead to Its Hallmark \\ Features \\ Systemic Inflammation and Inflammatory Markers: Diagnostic and \\ Prognostic Relevance \\ What Are Potential Targets for Intervention? \\ Summary
}

\begin{abstract}
Chronic critical illness (CCI) is syndrome of neuromuscular weakness, brain dysfunction, malnutrition, endocrinopathies, and symptom distress. Two conceptual themes may be useful in discussing CCI. The first is a clinical roadmap in which a patient suffers an acute critical illness, survives the initial insult, but yet is unable to be liberated from the ventilator. The second framework considers the effect of systemic inflammation and CCI, linking acute CCI risk factors with the common clinical features of CCI. Given the association between common CCI antecedents and inflammation, attempts to control and balance the pro-inflammatory and anti-inflammatory mediators should begin as early as possible and continue throughout the ICU stay. Since surrogate measures such as biomarkers often fail to predict the effect of interventions, the focus should be on the outcomes patients experience. As of now, providing evidence-based, high quality ICU management of patients at risk for CCI appears to be the best strategy of care. Key words: inflammation; chronic critical illness. [Respir Care 2012;57(6):859-864. (c) 2012 Daedalus Enterprises]
\end{abstract}

\section{Introduction}

Chronic critical illness $(\mathrm{CCI})$ is an important clinical phenomenon that all critical care and respiratory therapists recognize. $\mathrm{CCI}$ is a relatively common clinical entity and will continue to increase in incidence during the coming

\footnotetext{
Dr Cox is affiliated with the Division of Pulmonary, Allergy, and Critical Care Medicine, Duke University Medical Center, Durham, North Carolina.

Dr Cox presented a version of this paper at the 49th RESPIRATORY CARE Journal Conference, "The Chronically Critically Ill Patient," held September 9-10, 2011, in St Petersburg, Florida.
}

Dr Cox has disclosed a relationship with RTI International. decade. Part of the expansion of the numbers of CCI patients is likely driven by patient preferences, surrogate decision maker input, societal expectations, and providerfamily communication. However, the population is aging and more susceptible to critical illness with greater age. It is worth considering what factors may predispose patients to slower recovery or death in the context of critical illness.

Correspondence: Christopher E Cox MD MPH, Division of Pulmonary, Allergy, and Critical Care Medicine, Duke University Medical Center, DUMC 102043, Durham NC 27710. E-mail: christopher.cox@duke.edu.

DOI: $10.4187 /$ respcare.01719 


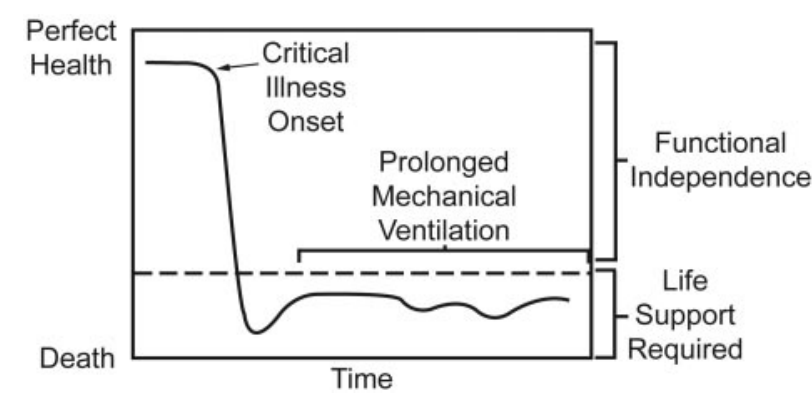

Fig. 1. Conceptual model of chronic critical illness. This figure shows chronic critical illness as an inability to be liberated from prolonged mechanical ventilation. Functional independence is compromised as the duration of life support increases.

There is evidence that the host systemic inflammatory response may mediate the course of critical illness, possibly contributing to the development of CCI. This review will review these data and highlight potential targets for future interventions designed to address this chronic inflammation.

\section{What Drives the Inflammatory Response in CCI?}

There are 2 conceptual models that may be useful in discussing CCI. The first is a clinical roadmap in which a patient suffers an acute critical illness, survives the initial insult, yet is unable to be liberated from life support (Fig. 1). In the past, much research has been focused on case definitions of CCI, the short- and long-term outcomes of these patients, and their resource utilization. ${ }^{1,2}$ It was not clear, however, what factors were most important in CCI patients' failure to recover in a timely fashion to the point where mechanical ventilation was no longer required.

During the past decade, a number of important studies have highlighted the potential mediating effect of illness severity, type, as well as management on critical care outcomes. Low tidal volume ventilation, daily awakenings from sedation, glucose control strategies, targeted use of corticosteroids, early goal directed therapy for sepsis, and early mobilization of critically ill patients have all demonstrated some effect on both mortality and morbidity. When subsequent research on the mechanisms of some of these interventions was done, there was often evidence of a differential host inflammatory response between intervention and control. For example, patients receiving lower tidal volumes have comparatively lower concentrations of interleukin 6 (IL-6) and tumor necrosis factor alpha (TNF- $\alpha$ ) in their bronchoalveolar lavage fluid than those receiving higher tidal volumes. ${ }^{3}$ Corticosteroids can augment anti-inflammatory responses in critical illness in some patients, though there are complicated and conflicting proinflammatory profiles induced. ${ }^{4}$
Although there are few studies that have focused specifically on CCI and the inflammatory response, generalizations and hypotheses might be useful to consider. A second helpful conceptual framework for considering the effect of systemic inflammation and CCI is the linkage of acute CCI precipitants with common clinical features of CCI (Fig. 2). One can see that acute critical illnesses such as acute lung injury, sepsis, and trauma may lead to CCI. These acute processes may be mediated by risk factors such as age, sex, illness severity and type, and management styles (eg, low tidal volumes in acute lung injury). However, the host inflammatory response-adequate or inadequate, pro-inflammatory versus anti-inflammatorymay moderate the transformation of an acute critical illness into a chronic condition. These inflammatory responses therefore could directly be related to the acquisition of ICU-related weakness, brain dysfunction, malnutrition, and other hallmark descriptors of CCI. Although this framework is conceptual only, it may be helpful for the respiratory therapist when considering intervention targets designed to mitigate the antecedents, symptoms, and signs of CCI.

\section{Clinical Features of Chronic Critical Illness and Their Relationship to Systemic Inflammation}

$\mathrm{CCI}$ is commonly characterized by stereotypical phenotype of tracheotomy placement and the requirement for prolonged mechanical ventilation, typically after at least 10 days of ventilation or more. Rather than categorization by a single procedure, perhaps a more accurate view of CCI relevant to future intervention planning is that of a syndrome of neuromuscular weakness, brain dysfunction, malnutrition, endocrinopathies, and symptom distress. ${ }^{2} \mathrm{Al}-$ though these manifestations of CCI are themselves interrelated in a complex fashion, the example of neuromuscular dysfunction (ie, ICU-acquired weakness) will be used later as an example of how systemic inflammation can lead to CCI.

\section{Inflammation, Inflammatory Markers, and CCI}

Common CCI antecedents including sepsis and acute lung injury have well characterized host immune system responses as well as both mediators and markers of systemic inflammation. Pro-inflammatory mediators in these conditions include TNF- $\alpha$, IL- 1 and IL-6, prostaglandins, leukotrienes, bradykinins, platelet-activating factor, proteases, oxidants, and nitric oxide. ${ }^{3,5}$ These mediators may lead to multisystem organ dysfunction and death when unchecked by anti-inflammatory mechanisms and mediators, including IL-4 and IL-10. Although past hypotheses have suggested an imbalance in pro- and anti-inflammatory cytokines or an overly exuberant cytokine-regulated, 


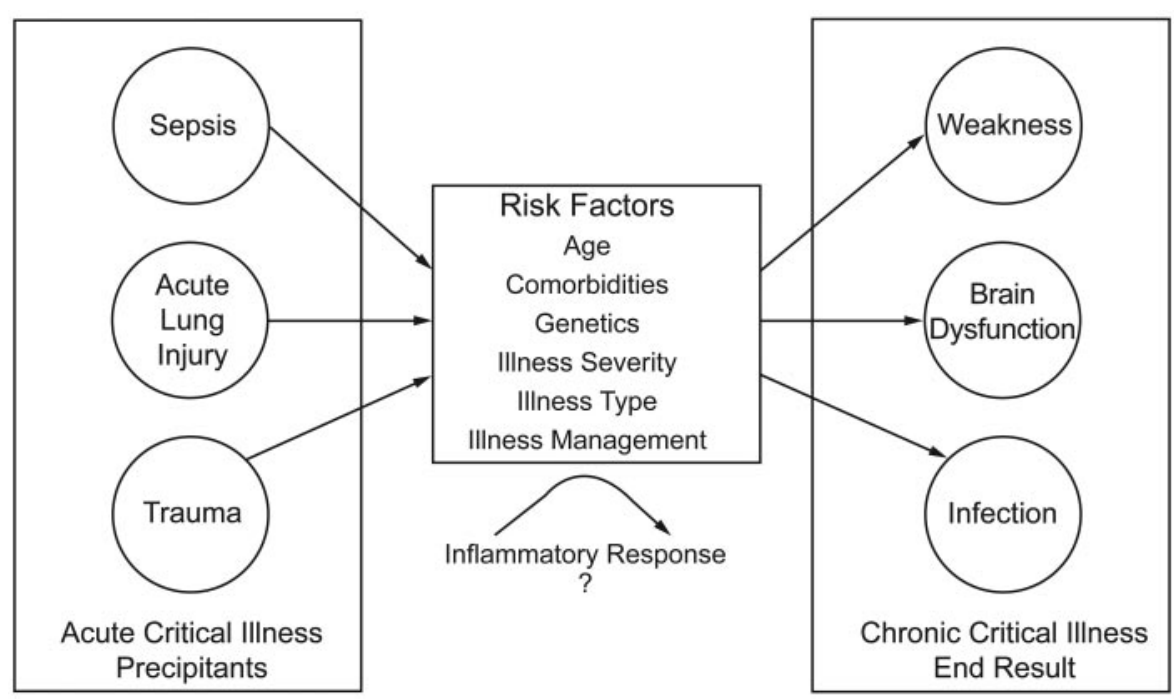

Fig. 2. Risk factors for chronic critical illness. Common precipitants of chronic critical illness, such as sepsis, acute lung injury, and trauma, may be mediated by risk factors shown in the figure. The activity of the host inflammatory response may mediate these risk factors, accentuating their impact on life support duration.

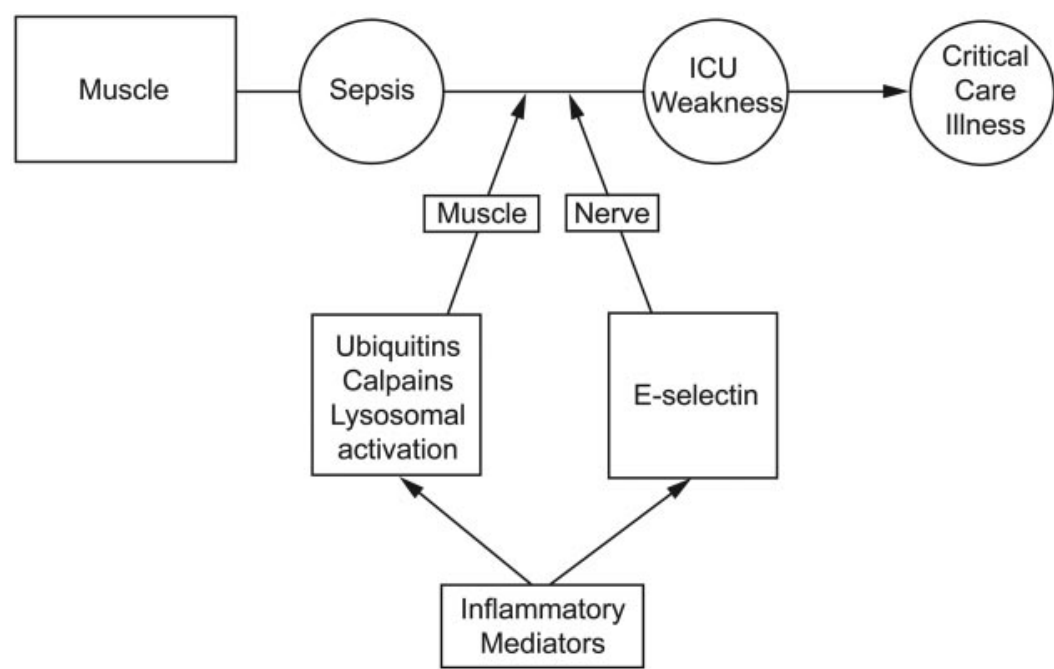

Fig. 3. Muscle weakness in chronic critical illness. Muscle weakness may be driven in part by the effect of sepsis-related inflammatory mediators on muscle and nerve tissue.

illness-associated immunosuppression that affects sepsis outcomes, in reality there is no clear "normal" response. General patterns that may be expected include an initial hyperinflammatory state, followed by a return to normal cytokine levels - though a return that may include a hypoinflammatory period. ${ }^{6}$

\section{Inflammation Seen in Precipitants of CCI May Lead to Its Hallmark Features}

Returning to the example of ICU-acquired weakness, it is useful to consider the example of septic shock in the development of CCI (Fig. 3). First, sepsis is strongly as- sociated with weakness in ICU patients. Among patients mechanically ventilated for a week or more, $50-75 \%$ have neurophysiologic testing abnormalities, ${ }^{7,8}$ and nearly all with septic shock have abnormalities on muscle biopsy, electromyography, and nerve conduction studies. ${ }^{9-11} \mathrm{Sec}-$ ond, muscle weakness is also associated with both survival $^{9}$ and prolonged mechanical ventilation. ${ }^{12,13}$ This muscle weakness is often profound and affects both peripheral muscles as well as the diaphragm itself, which appears to undergo muscle atrophy and contractile dysfunction as soon as after 18 hours of inactivity. ${ }^{14,15}$ Third, the inflammatory response has direct effect on muscles and nerves in the setting of serious illness. ${ }^{16}$ Critical illness myopathy is 
known to be associated with sodium channel abnormalities, mitochondrial dysfunction, glutathione depletion, and nitric oxide production. ${ }^{17}$ The proteolysis seen in critical illness myopathy is related to ubiquitins, calpains, and both lysosomal and non-lysosomal systems, themselves modulated in part by pro-inflammatory pathways. ${ }^{18-21}$ Critical illness associated polyneuropathy is associated with an increase in E-selectin in the epineurium and endoneurium, mediated by TNF- $\alpha$ and IL-1.22 This can lead to tissue injury and greater cytokine activation through endothelial cell leukocyte adhesion and increase in the number of activated leukocytes present in the endoneurium. ${ }^{23}$ Cytokine activation can also increase neurovascular permeability, thus allowing neurotoxins to enter and damage the nerve tissue itself. ${ }^{24}$

\section{Systemic Inflammation and Inflammatory Markers: Diagnostic and Prognostic Relevance}

Given this association between common CCI antecedents and inflammation, it is worthwhile to consider how the inflammatory response can be measured and these data used to diagnose, provide prognosis, and monitor therapy. Clinical markers of systemic inflammation such as temperature, respiratory rate, blood pressure, white-blood-cell count, and platelet count are simple to measure, but are non-specific. Indirect markers of inflammation such as platelet count, itself closely related to processes such as sepsis, are nonetheless compelling since they are a component of the ProVent mortality prediction model for prolonged mechanical ventilation, a highly accurate statistical tool. ${ }^{25,26}$ However, the best known and most widely available serum clinical markers of systemic inflammation include C-reactive protein (CRP) and procalcitonin. Although CRP and procalcitonin levels are themselves correlated in the setting of critical illness, ${ }^{27}$ they have different performance characteristics when used for diagnosis, response to therapy, and prognosis.

For diagnosis, CRP has demonstrated mixed findings. In the setting of sepsis, CRP has been shown to correlate with illness severity ${ }^{28,29}$ in some populations, though not in others. ${ }^{27,30}$ In populations with community-acquired pneumonia, CRP has also shown limited ability to predict illness severity. ${ }^{31}$ In general, the prognostic capability of CRP levels has been poor among seriously ill patients, owing to its low to moderate specificity, ranging from 40-85\%. 30,32,33 CRP monitoring fares better in assessing the response to therapy in populations including patients who have pancreatitis or who have sepsis. ${ }^{34}$ Also, CRP changes over time have been found to be associated with postoperative survival. ${ }^{35,36}$ Based on the current data, at this time the additional benefit of using CRP in a clinical setting is unclear.
Procalcitonin too has a mixed history in the setting of serious illness. In 2007, Tang et al reported the results of a systematic review and meta-analysis of procalcitonin gathered from 18 relevant studies. ${ }^{37}$ These investigators found that the sensitivity of procalcitonin for the diagnosis of sepsis was low (71\%), as was the area under the receiver operator characteristic curve (0.78). Since that time, others have reported somewhat better diagnostic accuracy for procalcitonin and sepsis. ${ }^{38}$ In lower-respiratory-tract infections, procalcitonin monitoring did not improve outcomes but reduced total antibiotic exposure. ${ }^{39}$ However, in other studies the benefits of procalcitonin-guided antibiotic strategies have been questioned. ${ }^{40}$ Longitudinal changes in procalcitonin may be associated with survival in septic shock and in the critically ill in general, though questions remain about the discriminatory strength of these assessments. ${ }^{27,41}$

However, the observations of Kellum and colleagues have illuminated the importance of temporal context with regard to inflammatory marker monitoring. In a cohort of 1,886 patients with severe sepsis and community-acquired pneumonia, these researchers found that inflammatory cytokines were highest at presentation in the emergency department and remained elevated beyond resolution of infection, while systemic inflammation was not uniformly observed..$^{42}$ They also found that there was no increase in inflammation seen after the onset of organ dysfunction. Importantly, elevated inflammatory markers, including IL-6 and TNF- $\alpha$, as well as anti-inflammatory markers such as IL-10, were seen commonly among both survivors and non-survivors.

\section{What Are Potential Targets for Intervention?}

Given the complexity, redundancy, and overlapping nature of the human innate immune system and the inflammatory response to critical illness, it is difficult to extend the data reviewed above to either explain $\mathrm{CCI}$ or direct its specific therapy. It appears that targeting specific single pro-inflammatory cytokines is not likely to be a successful strategy in this setting. ${ }^{5}$ However, recent work suggests that focusing on more general targets, such as in the case of oxidative stressors, may hold promise. ${ }^{43}$ But what is likely to be true in CCI is that attention must be given to modifying its risk factors, intervening early, and continuing therapy throughout the course of critical illness (Fig. 4).

Perhaps the most effective contemporary treatment pathway for clinicians who manage the critically ill can again be viewed in the example of ICU-acquired weakness, a risk factor for CCI. As described by Schweickert and Hall, anticipatory management, including risk factor modification for those likely to develop prolonged mechanical ventilation, is the best current management strategy. ${ }^{44} \mathrm{Com}-$ plementary management strategies such as daily interrup- 


\section{Persistent Systemic Inflammation in Chronic Critical Illness}

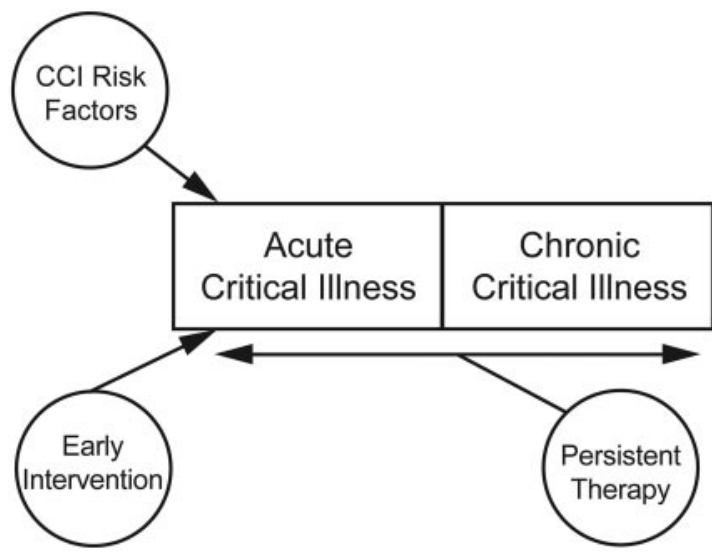

Fig. 4. Future template for chronic critical illness (CCl) therapy. Future research may be best applied to manage risk factors for prolonged life support early in the course of critical illness, continuing therapy beyond the first few days of critical care.

tion of sedatives, ventilation strategies that limit tidal volumes, and the use of spontaneous breathing trials can reduce the length of ventilation, promote recovery, and may reduce complications of prolonged critical illness such as muscle weakness. However, evolving interventions that seek to address weakness such as the early mobilization of patients are not only intuitive, but are supported by a substantial body of literature demonstrating that inactivity promotes inflammation and oxidative stressors that in turn worsen organ function. ${ }^{45}$ Mobilization of the critically ill patient can reduce circulating levels of pro-inflammatory cytokines. ${ }^{46}$ Overall, however, our current level of understanding of this clinical problem's diagnosis, prognosis, and treatment is informed by the associated inflammatory profile but is not substantially improved by the monitoring of systemic inflammation.

\section{Summary}

Chronic critical illness can be conceptualized as a clinical syndrome that may be moderated in part by systemic inflammation and the balance of pro- and anti-inflammatory mediators. Although neither the specific monitoring of the inflammatory process nor the targeting of specific inflammatory cytokines has shown unquestioned benefit, these practices may hold promise as targets for future research. The current medical literature suggests that inflammatory-mediator-based interventions should take into account CCI risk factors, be administered early in the course of critical illness, and be continued through the duration of ICU care. As of now, providing evidence-based, high quality ICU management of patients at risk for CCI appears to be the best strategy of care. The emphasis should remain focused on the outcomes patients experience, be- cause surrogate measures such as biomarkers often fail to predict the effect of interventions. ${ }^{47}$

\section{REFERENCES}

1. Carson SS, Bach PB. The epidemiology and costs of chronic critical illness. Crit Care Clin 2002;18(3):461-476.

2. Nelson JE, Cox CE, Hope AA, Carson SS. Chronic critical illness. Am J Respir Crit Care Med 2010;182(4):446-454.

3. Ranieri VM, Suter PM, Tortorella C, De Tullio R, Dayer JM, Brienza A, et al. Effect of mechanical ventilation on inflammatory mediators in patients with acute respiratory distress syndrome: a randomized controlled trial. JAMA 1999;282(1):54-61.

4. Marik PE. Critical illness-related corticosteroid insufficiency. Chest 2009;135(1):181-193.

5. Russell JA. Management of sepsis. N Engl J Med 2006;355(16): 1699-1713.

6. Hotchkiss RS, Karl IE. The pathophysiology and treatment of sepsis. N Engl J Med 2003;348(2):138-150.

7. van den Berghe G, Wouters P, Weekers F, Verwaest C, Bruyninckx $\mathrm{F}$, Schetz $\mathrm{M}$, et al. Intensive insulin therapy in the critically ill patients. N Engl J Med 2001;345(19):1359-1367.

8. Leijten FS, Harinck-de Weerd JE, Poortvliet DC, de Weerd AW. The role of polyneuropathy in motor convalescence after prolonged mechanical ventilation. JAMA 1995;274(15):1221-1225.

9. Garnacho-Montero J, Madrazo-Osuna J, Garcia-Garmendia JL, OrtizLeyba C, Jimenez-Jimenez FJ, Barrero-Almodovar A, et al. Critical illness polyneuropathy: risk factors and clinical consequences. A cohort study in septic patients. Intensive Care Med 2001;27(8):1288-1296.

10. Tepper M, Rakic S, Haas JA, Woittiez AJ. Incidence and onset of critical illness polyneuropathy in patients with septic shock. Neth J Med 2000;56(6):211-214.

11. Coakley JH, Nagendran K, Honavar M, Hinds CJ. Preliminary observations on the neuromuscular abnormalities in patients with organ failure and sepsis. Intensive Care Med 1993;19(6):323-328.

12. De Jonghe B, Sharshar T, Lefaucheur JP, Authier FJ, Durand-Zaleski I, Boussarsar M, et al. Paresis acquired in the intensive care unit: a prospective multicenter study. JAMA 2002;288(22):2859-2867.

13. Hough CL, Steinberg KP, Taylor Thompson B, Rubenfeld GD, Hudson LD. Intensive care unit-acquired neuromyopathy and corticosteroids in survivors of persistent ARDS. Intensive Care Med 2009;35(1):63-68.

14. Levine S, Nguyen T, Taylor N, Friscia ME, Budak MT, Rothenberg $\mathrm{P}$, et al. Rapid disuse atrophy of diaphragm fibers in mechanically ventilated humans. N Engl J Med 2008;358(13):1327-1335.

15. Jaber S, Petrof BJ, Jung B, Chanques G, Berthet JP, Rabuel C, et al. Rapidly progressive diaphragmatic weakness and injury during mechanical ventilation in humans. Am J Respir Crit Care Med 2011; 183(3):364-371.

16. de Letter MA, Schmitz PI, Visser LH, Verheul FA, Schellens RL, Op de Coul DA, et al. Risk factors for the development of polyneuropathy and myopathy in critically ill patients. Crit Care Med 2001; 29(12):2281-2286.

17. Brealey D, Brand M, Hargreaves I, Heales S, Land J, Smolenski R, et al. Association between mitochondrial dysfunction and severity and outcome of septic shock. Lancet 2002;360(9328):219-223.

18. Di Giovanni S, Molon A, Broccolini A, Melcon G, Mirabella M, Hoffman EP, et al. Constitutive activation of MAPK cascade in acute quadriplegic myopathy. Ann Neurol 2004;55(2):195-206.

19. Showalter CJ, Engel AG. Acute quadriplegic myopathy: analysis of myosin isoforms and evidence for calpain-mediated proteolysis. Muscle Nerve 1997;20(3):316-322.

20. Tiao G, Hobler S, Wang JJ, Meyer TA, Luchette FA, Fischer JE, et al. Sepsis is associated with increased mRNAs of the ubiquitin- 


\section{Persistent Systemic Inflammation in Chronic Critical Illness}

proteasome proteolytic pathway in human skeletal muscle. J Clin Invest 1997;99(2):163-168

21. Mitch WE, Goldberg AL. Mechanisms of muscle wasting. The role of the ubiquitin-proteasome pathway. N Engl J Med 1996;335(25): 1897-1905.

22. Fenzi F, Latronico N, Refatti N, Rizzuto N. Enhanced expression of E-selectin on the vascular endothelium of peripheral nerve in critically ill patients with neuromuscular disorders. Acta Neuropathol 2003;106(1):75-82.

23. Latronico N, Peli E, Botteri M. Critical illness myopathy and neuropathy. Curr Opin Crit Care 2005;11(2):126-132.

24. Druschky A, Herkert M, Radespiel-Troger M, Druschky K, Hund E, Becker CM, et al. Critical illness polyneuropathy: clinical findings and cell culture assay of neurotoxicity assessed by a prospective study. Intensive Care Med 2001;27(4):686-693.

25. Katz JN, Kolappa KP, Becker RC. Beyond thrombosis: the versatile platelet in critical illness. Chest 2011;139(3):658-668.

26. Carson SS, Cox CE, Holmes GM, Howard A, Carey TS. The changing epidemiology of mechanical ventilation: a population-based study. J Intensive Care Med 2006;21(3):173-182.

27. Claeys R, Vinken S, Spapen H, ver Elst K, Decochez K, Huyghens $\mathrm{L}$, et al. Plasma procalcitonin and $\mathrm{C}$-reactive protein in acute septic shock: clinical and biological correlates. Crit Care Med 2002;30(4): 757-762.

28. Povoa P, Almeida E, Moreira P, Fernandes A, Mealha R, Aragao A, et al. C-reactive protein as an indicator of sepsis. Intensive Care Med 1998;24(10):1052-1056.

29. Lobo SM, Lobo FR, Bota DP, Lopes-Ferreira F, Soliman HM, Melot $\mathrm{C}$, et al. C-reactive protein levels correlate with mortality and organ failure in critically ill patients. Chest 2003;123(6):2043-2049.

30. Silvestre J, Povoa P, Coelho L, Almeida E, Moreira P, Fernandes A, et al. Is C-reactive protein a good prognostic marker in septic patients? Intensive Care Med 2009;35(5):909-913.

31. Muller B, Harbarth S, Stolz D, Bingisser R, Mueller C, Leuppi J, et al. Diagnostic and prognostic accuracy of clinical and laboratory parameters in community-acquired pneumonia. BMC Infect Dis 2007; 7:10.

32. Pettila V, Hynninen M, Takkunen O, Kuusela P, Valtonen M. Predictive value of procalcitonin and interleukin 6 in critically ill patients with suspected sepsis. Intensive Care Med 2002;28(9):12201225.

33. Povoa P. C-reactive protein: a valuable marker of sepsis. Intensive Care Med 2002;28(3):235-243.

34. Luzzani A, Polati E, Dorizzi R, Rungatscher A, Pavan R, Merlini A. Comparison of procalcitonin and $\mathrm{C}$-reactive protein as markers of sepsis. Crit Care Med 2003;31(6):1737-1741.
35. Aouifi A, Piriou V, Bastien O, Blanc P, Bouvier H, Evans R, et al. Usefulness of procalcitonin for diagnosis of infection in cardiac surgical patients. Crit Care Med 2000;28(9):3171-3176.

36. Icard P, Fleury JP, Regnard JF, Libert JM, Magdeleinat P, Gharbi N, et al. Utility of C-reactive protein measurements for empyema diagnosis after pneumonectomy. Ann Thorac Surg 1994;57(4):933936.

37. Tang BM, Eslick GD, Craig JC, McLean AS. Accuracy of procalcitonin for sepsis diagnosis in critically ill patients: systematic review and meta-analysis. Lancet Infect Dis 2007;7(3):210-217.

38. Rowther FB, Rodrigues CS, Deshmukh MS, Kapadia FN, Hegde A, Mehta AP, et al. Prospective comparison of eubacterial PCR and measurement of procalcitonin levels with blood culture for diagnosing septicemia in intensive care unit patients. J Clin Microbiol 2009; 47(9):2964-2969.

39. Schuetz P, Christ-Crain M, Thomann R, Falconnier C, Wolbers M, Widmer I, et al. Effect of procalcitonin-based guidelines vs standard guidelines on antibiotic use in lower respiratory tract infections: the ProHOSP randomized controlled trial. JAMA 2009;302(10):1059-1066

40. Jensen JU, Hein L, Lundgren B, Bestle MH, Mohr TT, Andersen $\mathrm{MH}$, et al. Procalcitonin-guided interventions against infections to increase early appropriate antibiotics and improve survival in the intensive care unit: a randomized trial. Crit Care Med 2011;39(9): 2048-2058.

41. Jensen JU, Heslet L, Jensen TH, Espersen K, Steffensen P, Tvede M. Procalcitonin increase in early identification of critically ill patients at high risk of mortality. Crit Care Med 2006;34(10):2596-2602.

42. Kellum JA, Kong L, Fink MP, Weissfeld LA, Yealy DM, Pinsky MR, et al. Understanding the inflammatory cytokine response in pneumonia and sepsis: results of the Genetic and Inflammatory Markers of Sepsis (GenIMS) Study. Arch Intern Med 2007;167(15):1655-1663.

43. Powers SK, Hudson MB, Nelson WB, Talbert EE, Min K, Szeto HH, et al. Mitochondria-targeted antioxidants protect against mechanical ventilation-induced diaphragm weakness. Crit Care Med 2011;39(7): 1749-1759.

44. Schweickert WD, Hall J. ICU-acquired weakness. Chest 2007;131(5): 1541-1549.

45. Winkelman C. Inactivity and inflammation in the critically ill patient. Crit Care Clin 2007;23(1):21-34.

46. Winkelman C, Higgins PA, Chen YJ, Levine AD. Cytokines in chronically critically ill patients after activity and rest. Biol Res Nurs 2007;8(4):261-271.

47. Micheel CM, Ball JR. Evaluation of biomarkers and surrogate endpoints in chronic disease. Washington, DC: Institute of Medicine/ National Academies Press; 2010. http://www.nap.edu/openbook.php? isbn $=0309151295$.

\section{Discussion}

MacIntyre: The mechanisms underlying chronic critical illness are still a morass to me. I think that's the state of the art. It's a very challenging field.

White: The area of repair continues to evolve. There is some work now beginning in this area to do with progenitor endothelial cells that need to be mobilized from the bone marrow. We know that low platelets, for instance, is one of the predictors of poor outcome in some patients with CCI. What is that telling us about the role of the hematological system and recovery? The other area that is going to be of interest is mitochondrial function, and the ability to turn back on mitochondrial function after it's been shut down by septic shock is going to be important.

Cox: We have a number of colleagues at Duke who work in mitochondrial science who've been telling us this for some time, and I agree, it seems like a particularly promising area. In sepsis the mitochondria are literally exploding, which is clearly problematic for the musculature. The 


\section{Persistent Systemic Inflammation in Chronic Critical Illness}

data in this area are so strong that we will certainly be seeing more about it in the future.

Muldoon:* How related are the two questions of the role of inflammation in CCI and the recurrent question of what's the role of inflammation, meaning treatment with steroids, in severe sepsis?

Cox: That is a challenging, complicated question. Steroids can affect so many physiological pathways in both good ways and bad ways. Let me start by attempting to summarize what seems to work in the context of critical care: we're not sure! It seems that it's really unclear that there is a role for steroids in some of the most common illnesses that are also precipitants of CCI such as sepsis, acute lung injury, and pneumonia. A counter-argument would be that we haven't identified a true signal of benefit, mainly because we don't understand the subgroups of disease phenotypes. So I think we don't have persuasive data that demonstrates broad clinical benefit for steroids in critical illness or the prevention of CCI. Certainly, I am very concerned with the myopathy that could result from steroids, which might then increase the risk of CCI development, but that is my bias.

Girard: Regarding Sean Muldoon's question about the inflammatory process in sepsis and how it might inform us regarding inflammation in CCI as well as what Shannon Carson mentioned-which was that we may need to develop other ways of identifying patients with CCI in terms of biomarkers and so forth-made me realize that even though sepsis has been studied for about 30 years and we have a pretty good grasp of what happens in terms of inflammation in sepsis, we still use

\footnotetext{
* Sean R Muldoon MD MPH, Kindred Healthcare, Hospital Division, Louisville, Kentucky.
}

a very imperfect, clinical based definition to identify sepsis. We still don't have a biomarker to identify sepsis, as we do to identify myocardial infarction, for example. Can we develop a panel of biomarkers to identify CCI?

Cox: It would be nice, I think we all agree, to augment our prognostic capability with biological data.

Carson: I don't think we're going to come up with a magic biomarker of CCI. We have seen patients presenting with an acute inflammatory process pneumonia, and some were more severe than others, and often inflammation improved as the patients improved. However, some of those patients did not improve. They developed severe pneumonia or severe ARDS, and the ARDS was slow to recover and they became CCI. So what was the pattern of the inflammatory mediators in those patients? And did that pattern differ substantially from those who did recover quickly? It's an open question.

Alex White is on to something important in that these patients are different phenotypically, and therefore something biological is going on. If we could just find the right biomarkers, we might get an array of things that are different about these patients than those who recover and those who go on to CCI.

Nelson: Before his talk, I commented to Chris Cox that he had the hardest assignment in the room. I think he did a yeoman's job. Some interesting concepts have been proposed over the years, like "immunological exhaustion" after prolonged critical illness, but there hasn't been any research on this in the context of CCI, and much remains to be studied, even in the area of acute critical illness. The greatest challenge in caring for the chronically critically ill is the recurrent infections, to which these patients eventually succumb. The question really is, why hasn't there been enough research to help us address this challenge?

As a group, we need to think a lot about that, and ways to promote the research. I think it's an extremely high priority, both in acute and chronic critical illness. Until that happens, we're going to be struggling, but for now we can at least try to identify the questions and lobby for support to find some of the answers.

MacIntyre: I'm going to put on a slightly different hat. I' $m$ in the COPD Gene project, and they're getting right to the very issue of the genetic factors in COPD phenotypes. Specifically, in COPD, why is it that only $40 \%$ of people who pump noxious substances into their lungs develop chronic inflammation and the other $60 \%$ do not? There's got to be something fundamental in the genetic structure of smokers without COPD that produces a defense system that works really well. Genomics goes to the very fundamental structure of how cells behave. I think studying the inflammatory mediators is just scratching the surface.

Mechanick: The way that I'm putting this together in my mind is that we have this tension where it's difficult to attribute one or two biomarkers or cytokines to identify disease through any process of risk stratification. At the same time, we have a subjective fear of complexity. I would offer the following reconciliation of those points.

First of all, if you take a disease like Graves disease, a natural disease, you have a single marker: TSH [thyroid-stimulating hormone] receptor antibodies. So we can differentiate natural diseases from iatrogenesis. I think $\mathrm{CCI}$ is not a natural disease; rather, it is an iatrogenic disease. So, instead of looking at a component biology single gene marker, what we really need to look at is a systems biology genomic or transcriptonomic state. Since this process is iatrogenic and not natural 
per se, there's no Darwinian precedent: this state has not resulted from natural selection.

Once you do the biocomputational analysis of that very complex state, you may see emerging motifs and patterns with appropriate biomarkers around which you can design interventions. So, rather than looking at complexity philosophically as something we do not understand or subjectively fear, we should embrace it as a computational marker of the state of the patient at, say, day 23 in the ICU. This is not a natural condition: you're not going to find a single cytokine or a cadre of 7 cytokines that give a good predictive model with clean data.
White: I am not sure CCI is completely iatrogenic.

Mechanick: There would be no CCI state if there were no intervention for the acute critical illness state; the patient would have died without that intervention and never reached CCI, so $\mathrm{CCI}$ is indeed an iatrogenic state.

This article is approved for Continuing Respiratory Care Education credit. For information and to obtain your CRCE

(free to AARC members) visit

www.RCJournal.com 